\title{
Sets that are connected in two random graphs
}

\author{
Michael Molloy*
}

August 17, 2012

\begin{abstract}
We consider two random graphs $G_{1}, G_{2}$, both on the same vertex set. We ask whether there is a non-trivial set of vertices $S$, so that $S$ induces a connected subgraph both in $G_{1}$ and in $G_{2}$. We determine the threshold for the appearance of such a subset, as well as the size of the largest such subset.
\end{abstract}

\section{Introduction}

The giant component dates back to the earliest days of random graph theory[5] and still remains as one of the most studied phenomena in the field. In this paper, we consider a very natural variation on the giant component which, somewhat surprisingly, does not appear to have been studied before: giant vertex sets that are connected in two different random graphs, simultaneously.

Consider two random graphs, $G_{1}, G_{2}$, each drawn from the model $G_{n, p=c / n}$ for some constant $c>1$, and each on the same vertex set, $V=\{1, \ldots, n\}$. We say that $S \subseteq V$ is a doubly connected set of $\left(G_{1}, G_{2}\right)$ if $S$ induces a connected subgraph in $G_{1}$ and $S$ induces a connected subgraph in $G_{2}$. We consider a doubly connected set to be trivial if it has size at most 2 .

A simple argument shows that w.h.p. ${ }^{1}$ a non-trivial doubly connected set $S$ must lie in the intersection of the giant components of $G_{1}$ and $G_{2}$ (this follows, eg, from Lemma 3.1 below). It does not take long to realize that their intersection is w.h.p. not doubly connected. Indeed, it will contain a linear number of vertices which were, e.g. connected to the giant component of $G_{1}$ through a path of vertices that are not all in the giant component of $G_{2}$. At first thought, it is not clear whether there will be a non-trivial doubly connected set at all, even for very large constant $c$. In this paper, we show that there is. We determine the threshold for the appearance of a non-trivial doubly connected set, and we determine its size.

Define

$$
c^{*}=\min _{\xi>0} \frac{\xi}{\left(1-e^{-\xi}\right)^{2}}=2.4554 \ldots
$$

*Dept of Computer Science, University of Toronto, molloy@cs.toronto.edu. Research supported by an NSERC Discovery Grant.

${ }^{1}$ We say that a property holds w.h.p. (with high probability) if the probability tends to 1 as $n$ grows. 
and define $\alpha=\alpha(c)$ to be the greatest solution to

$$
\alpha=\left(1-e^{-\alpha c}\right)^{2} .
$$

Substituting $\xi=\alpha c$, we obtain $c=\xi /\left(1-e^{-\xi}\right)^{2}$ and so $\alpha(c)>0$ iff $c>c^{*}$.

Theorem 1.1 (a) For any $c<c^{*}$, w.h.p. $\left(G_{1}, G_{2}\right)$ does not have a doubly connected set of size greater than 2.

(b) For any $c>c^{*}$, w.h.p. $\left(G_{1}, G_{2}\right)$ has a doubly connected set, and the largest such set has size $\alpha(c) n+o(n)$.

At $c=c^{*}, \alpha=.5116 \ldots$ and $\alpha$ increases with $c$. So for $c>c^{*}$, w.h.p. the largest doubly connected set is on more than half the vertices. In comparison, the giant component of $G_{n, p=c^{*} / n}$ has size roughly $.8866 n$ and squaring tells us that the intersection of the giant components of $G_{1}, G_{2}$ would have size roughly $.786 n$.

Remark: A doubly connected set of size 2 is an edge that is selected for both graphs. The expected number of such edges is $c^{2} / 2$, and a straightforward Method of Moments argument (see e.g. [8]) shows that the number is asymptotically distributed like a Poisson variable. Thus, for $c<c^{*}$, the probability that there is a doubly connected set of size 2 is $1-e^{-c^{2} / 2}+o(1)$.

Our proof extends to several copies of $G_{n, p}$ with varying edge-probabilities. Consider random graphs $G_{1}, \ldots, G_{t}$, on the same vertex set, where $G_{i}$ is from $G_{n, p=c_{i} / n}$. If there is a solution to:

$$
\alpha=\prod_{i=1}^{t}\left(1-e^{-c_{i} \alpha}\right),
$$

then w.h.p. there is a set $S$ of size $\alpha n+o(n)$ such that $S$ induces a connected subgraph in each of $G_{1}, \ldots, G_{t}$. If there is no such solution, then w.h.p. there is no such $S$ on more than two vertices (in fact, on more than one vertex if $t \geq 3$ ). We omit the details of the proof adaptation.

The proof of our theorem will be reminiscent of studies of the $k$-core, the pure literal rule, and other similar problems $[14,11,6,7,9]$. There, one repeatedly removes vertices (literals, etc.) that have small degree, until a core remains. Here, we will repeatedly remove vertices that lie in small components of at least one of the two graphs. Analysis of the $k$-core process is enabled by the fact that, at each iteration, what remains is a random graph conditioned on certain degree sequence properties, which we know how to analyze. In our process, at each iteration, we find that what remains is, roughly, the giant component of a random graph on a smaller vertex set. This enables us to continue to analyze it.

The techniques developed in this paper are applied in [12], where we need to analyze a similar structure on the union of random bipartite graphs. In that setting, we have vertex sets $A_{1}, \ldots, A_{k}$ and a random bipartite graph on each pair $\left(A_{i}, A_{j}\right)$. The freezing threshold for $k$-colourings of $G_{n, p}$ (see [12] for a definition) is determined via the threshold for the appearance of sets $S_{i} \subset A_{i}$ such that for each $i, j$, the bipartite subgraph induced by $\left(S_{i}, S_{j}\right)$ is connected.

\section{Some intuition}

We begin with a short intuitive explanation for $\alpha$ and $c^{*}$. 
Lemma 3.1, below, shows that any non-trivial doubly connected set of $\left(G_{1}, G_{2}\right)$ must have linear size. So we can determine whether such a set exists by using an iterative stripping process. At each step, every vertex that is in a small (i.e. sublinear) component of either graph is removed from both graphs. We will show that w.h.p., at each stage each graph contains at most one component that is not small (i.e. the giant component). Lemma 3.1 implies that w.h.p. if this process removes all vertices then there is no doubly connected set; otherwise, the vertices that remain form the largest doubly connected set.

Let $\Theta_{1}, \Theta_{2}$ be the vertices that are removed, at any point during the procedure, because at the time of removal they lay in small components of $G_{1}, G_{2}$, respectively. Thus, the doubly connected component that we find is $S=V \backslash\left(\Theta_{1} \cup \Theta_{2}\right)$. Note that $S$ is the giant component of $G_{1} \backslash \Theta_{2}$ (the graph remaining after removing $\Theta_{2}$ from $G_{1}$ ) and is also the giant component of $G_{2} \backslash \Theta_{1}$.

Now make a leap of faith and suppose that, somehow, $G_{1}$ and $G_{2}$ come up with $\Theta_{1}, \Theta_{2}$ independently.

Suppose $\left|\Theta_{1}\right|=(1-\rho) n+o(n)$ for some constant $\rho>0$; by symmetry, it is reasonable to assume that we also have $\left|\Theta_{2}\right|=(1-\rho) n+o(n)$. S is the vertex-set of the giant component of $G_{1} \backslash \Theta_{2}$, and also of the giant component of $G_{2} \backslash \Theta_{1}$. Since $\Theta_{2}$ is independent of $G_{1}$, we can treat $G_{1} \backslash \Theta_{2}$ as $G_{n^{\prime}, p=c / n}$ where $n^{\prime}=n-\left|\Theta_{2}\right|=\rho n+o(n)$. So by Lemma 3.4 (below), $|S|=\alpha n+o(n)$ where

$$
\alpha=\rho\left(1-e^{-\alpha c}\right) .
$$

By the independence of $\Theta_{1}, \Theta_{2}$, our leap of faith can also lead us to assume that $\Theta_{1}$ intersects $V \backslash \Theta_{2}$ in the same proportion that it intersects $V . \Theta_{1} \backslash \Theta_{2}$ is simply the set of vertices not appearing on the giant component of $G_{1} \backslash \Theta_{2}$, and so

$$
\frac{\left|\Theta_{1} \backslash \Theta_{2}\right|}{\left|V \backslash \Theta_{2}\right|}=\frac{\left|\Theta_{1}\right|}{|V|} \quad \rightarrow \quad \frac{\rho-\alpha}{\rho}=1-\rho \quad \rightarrow \quad \alpha=\rho^{2} .
$$

(1) and (2) yield $\alpha=\left(1-e^{-\alpha c}\right)^{2}$ and hence the definition of $c^{*}$.

Of course, in our process, $\Theta_{1}, \Theta_{2}$ are not formed independently. However, our proof can be viewed as building a different pair of sets $\Theta_{1}^{\prime}, \Theta_{2}^{\prime}$ with $\Theta_{i} \subseteq \Theta_{i}^{\prime}$, which yields the same doubly connected set; i.e. where $\Theta_{1} \cup \Theta_{2}=\Theta_{1}^{\prime} \cup \Theta_{2}^{\prime}$ and $S=V \backslash\left(\Theta_{1}^{\prime} \cup \Theta_{2}^{\prime}\right)$ is the giant component of both $G_{1} \backslash \Theta_{2}^{\prime}$ and $G_{2} \backslash \Theta_{1}^{\prime}$. Furthermore, $\Theta_{1}^{\prime}, \Theta_{2}^{\prime}$ will be formed (essentially) independently, enabling an analysis similar to that above.

\section{$3 \quad$ A stripping procedure}

We start by proving that w.h.p. every doubly connected set with more than 2 vertices must have linear size.

Lemma 3.1 For every $c>0$, there exists a constant $\phi=\phi(c)>0$ such that w.h.p. $\left(G_{1}, G_{2}\right)$ does not have a doubly connected set of size greater than 2 and less than $\phi n$.

Proof This is a very standard argument, using the principle that w.h.p. the subgraph induced by any small set of vertices must have very low edge-density. 
Let $X_{a}$ denote the number of doubly connected sets of size $a$. Each such set must contain a spanning tree in $G_{1}$ and in $G_{2}$. There are $a^{a-2}$ spanning trees on $a$ vertices, and each has exactly $a-1$ edges. So:

$$
E\left(X_{a}\right) \leq\left(\begin{array}{l}
n \\
a
\end{array}\right)\left(a^{a-2}\right)^{2}\left(\frac{c}{n}\right)^{2 a-2}<\frac{n^{2}}{a^{4} c^{2}}\left(\frac{e n}{a} a^{2}\left(\frac{c}{n}\right)^{2}\right)^{a}<\frac{n^{2}}{a^{4} c^{2}}\left(\frac{e c^{2} a}{n}\right)^{a} .
$$

From this, it is straightforward to obtain $\sum_{a=3}^{\phi n} E\left(X_{a}\right)=o(1)$ for $\phi<\frac{1}{e c^{2}}$.

Lemma 3.1 implies that any doubly connected set of size at least 3 must be contained in the intersection of the giant components of $G_{1}$ and $G_{2}$. Furthermore, the following procedure will w.h.p. find it.

At each iteration $i$, we will have a set of vertices $V_{i} \subset\{1, \ldots, n\}$. We define $S_{i}$, resp. $T_{i}$, to be the vertex set of the largest component of the subgraph of $G_{1}$, resp. $G_{2}$, induced by $V_{i}$.

\section{STRIP:}

Initialize $V_{1}=\{1, \ldots, n\}$.

For $i=1$ to $\infty$

Expose the vertices of $S_{i}$.

Expose the vertices of $T_{i}$.

if $S_{i}=T_{i}=V_{i}$ then HALT SUCCEED.

else

$V_{i+1}:=S_{i} \cap T_{i}$.

if $V_{i+1}=\emptyset$ then HALT FAIL.

To clarify: at each iteration, we only expose the vertices in $S_{i}, T_{i}$; we do not expose any of the edges amongst those vertices. This will be important for our analysis (see e.g. Observation 4.1). Informally, suppose that an oracle sees all of $G_{1}$ and $G_{2}$, and at each iteration, only tells us which vertices are in $S_{i}, T_{i}$; any probabilistic analysis that we carry out is conditioned on the information that the oracle has given us thus far. Note that the information the oracle provides is enough for us to carry out the procedure.

More formally, $S_{i} \subset V_{i}$ is a random set of vertices, such that the probability that $S_{i}=S$ is the probability that $S$ is the vertex set of the largest component of $G_{1}$ induced by $V_{i}$; after $S_{i}=S$ is selected we then condition all remaining random choices on the event that that vertex set is equal to $S$. (And similarly for $T_{i}$.)

We will prove that w.h.p.: If we halt Fail, then there is no doubly connected set of linear size. If we halt Succeed then $S_{i}=T_{i}$ is doubly connected, and is the maximum doubly connected set in $\left(G_{1}, G_{2}\right)$.

This is the iterative procedure described in Section 2. If it halts Succeed, then $\Theta_{1}, \Theta_{2}$ are $\cup_{i \geq 1} V_{i} \backslash S_{i}, \cup_{i \geq 1} V_{i} \backslash T_{i}$.

To analyse STRIP, we define:

$$
\rho_{1}=1
$$

for $i \geq 1, \gamma_{i}$ is the greatest solution to

$$
\gamma_{i}=\rho_{i}\left(1-e^{-\gamma_{i} c}\right),
$$


and

$$
\rho_{i+1}=\gamma_{i} / \rho_{i}=1-e^{-\gamma_{i} c}
$$

Note: If $\rho_{i} \leq 1 / c$ then $\gamma_{i}=0$, and if $\rho_{i}>1 / c$ then there is only one positive solution to (3).

Lemma 3.2 The sequences $\gamma_{1}, \ldots$ and $\rho_{1}, \ldots$ are both strictly decreasing and

(a) For any $c<c^{*}$, there exists $I$ such that $\gamma_{I}=\rho_{I}=0$.

(b) For any $c>c^{*}, \lim _{i \rightarrow \infty} \gamma_{i}=\alpha(c)$ and $\lim _{i \rightarrow \infty} \rho_{i}=\sqrt{\alpha(c)}$.

Proof It is easy to confirm that $\rho_{2}<1=\rho_{1}$. Suppose $\rho_{i}<\rho_{i-1}$. Then it is straightforward to check that $\gamma_{i}<\gamma_{i-1}$ (in fact, the proof of Lemma 3.4 below implies this). Note that $\rho_{i+1}=$ $1-e^{-\gamma_{i} c}$ and so $\gamma_{i}<\gamma_{i-1}$ implies $\rho_{i+1}<\rho_{i}$. So both sequences are decreasing. Since they are both positive, they have a limit. That limit must be a fixed point of the recursive equations. At that fixed point $(\rho, \gamma)$, we have $\rho=\gamma / \rho$ and so $\gamma=\rho^{2}$. This yields:

$$
\rho=1-e^{-c \rho^{2}}
$$

For $c<c^{*}$ the only solution to (4) is $\rho=0$, and so $\rho_{i}, \gamma_{i}$ tend to 0 . Therefore, there exists $I$ such that $\rho_{I-1} \leq 1 / c$ and hence $\gamma_{I-1}=\rho_{I}=\gamma_{I}=0$.

For $c \geq c^{*}$, the largest solution to (4) is $\rho=\sqrt{\alpha}$. A simple induction shows that $\rho_{i} \geq \sqrt{\alpha}$ for each $i$, and this yields part (b). Indeed, define $f(x)=1-e^{-y c}$ where $y=y(x)$ is the greatest solution to $y=x\left(1-e^{-y c}\right)$. Thus $\rho_{i+1}=f\left(\rho_{i}\right)$. Now $y(x)$ is non-decreasing and hence so is $f(x)$. Also note that $f(\sqrt{\alpha})=\sqrt{\alpha}$. So if $\rho_{i} \geq \sqrt{\alpha}$ then $\rho_{i+1}=f\left(\rho_{i}\right) \geq f(\sqrt{\alpha})=\sqrt{\alpha}$. Since $\rho_{1}=1>\sqrt{\alpha}$, this completes the induction.

In the next section, we will prove:

Lemma 3.3 For any constant $i$, and any constant $\phi>0$, w.h.p.

(a) $\left|V_{i}\right|=\rho_{i}^{2} n+o(n)$;

(b) $\left|S_{i}\right|,\left|T_{i}\right|=\gamma_{i} n+o(n)$;

(c) every vertex in $V_{i} \backslash V_{i+1}$ lies in a component of size less than $\phi$ n in either $G_{1} \cap V_{i}$ or $G_{2} \cap V_{i}$.

Lemmas 3.2, 3.3 show us that, by taking a sufficiently high constant number of iterations, $S_{i}, T_{i}$ get quite close to what Theorem 1.1 would predict. Lemma 3.3(c) and Lemma 3.1 imply inductively that every vertex not in $V_{i}$ does not lie in a non-trivial doubly connected set. Lemma 3.1 completes the proof of Theorem 1.1(a), by considering $i$ high enough so that $\gamma_{i}<\phi$. The proof of Theorem 1.1(b) requires more work.

We close this section with some useful facts about the size of a giant component. We say $X \in Y \pm Z$ to mean that $X$ is in the range $[Y-Z, Y+Z]$.

Lemma 3.4 For $c \tau>1$, w.h.p. the size of the largest component of $G_{n^{\prime}, p=c / n}$ where $n^{\prime}=\tau n$ is in $\beta n \pm n^{3 / 5}$, where $\beta$ is the positive solution to $\beta=\tau\left(1-e^{-\beta c}\right)$. 
Proof Note that $G_{n^{\prime}, p=c / n}$ is $G_{n^{\prime}, p=c \tau / n^{\prime}}$. So the classical result by Erdös and Rényi[5] on the size of the giant component in $G_{n, p}$, along with the fact that the distribution of the size is asymptotically normal $[16,15,3]$ implies that w.h.p. the size of the largest component is in $b n^{\prime} \pm n^{3 / 5}$ where $b$ is the positive solution to $b=1-e^{-c \tau b}$. The lemma follows by noting that $\beta=b \tau$.

We are interested in how $\beta$ changes with $\tau$ near $\tau=\sqrt{\alpha}$.

Lemma 3.5 For every $c>c^{*}$, at $\tau=\sqrt{\alpha}$ we have

$$
\frac{\partial}{\partial \tau} \beta<2 \sqrt{\alpha}
$$

Proof We use $\beta^{\prime}$ to denote $\frac{\partial}{\partial \tau} \beta$. So

$$
\beta^{\prime}=\left(1-e^{-\beta c}\right)+\tau \frac{\partial}{\partial \tau}\left(1-e^{-\beta c}\right)=\left(1-e^{-\beta c}\right)+\tau c e^{-\beta c} \beta^{\prime} .
$$

Solving for $\beta^{\prime}$ yields:

$$
\beta^{\prime}=\frac{1-e^{-\beta c}}{1-\tau c e^{-\beta c}}
$$

Recall that $c^{*}$ is a minimum of $\frac{\xi}{\left(1-e^{-\xi}\right)^{2}}$. It is easy to check that $\frac{\xi}{\left(1-e^{-\xi}\right)^{2}}$ is increasing above $c^{*}$ and so, since $c>c^{*}$, the derivative with respect to $\xi$ is postive. Differentiating and simplifying yields:

$$
1-e^{-\xi}>2 \xi e^{-\xi}
$$

Applying $\xi=\alpha c$, and $\sqrt{\alpha}=1-e^{-\alpha c}$, this yields $\sqrt{\alpha}>2 \alpha c e^{-\alpha c}$ so $\frac{1}{2}>\sqrt{\alpha} c e^{-\alpha c}$. Noting that at $\tau=\sqrt{\alpha}$ we have $\beta=\alpha$, yields that at $\tau=\sqrt{\alpha}$ :

$$
\frac{\partial}{\partial \tau} \beta=\frac{1-e^{-\alpha c}}{1-\sqrt{\alpha} c e^{-\alpha c}}<\frac{\sqrt{\alpha}}{1-\frac{1}{2}}=2 \sqrt{\alpha} .
$$

We close this section with the Chernoff Bound[4]. We use the version presented in [13]. Here, $B I N(n, p)$ is the sum of $n$ independent variables, each equal to 1 with probability $p$ and 0 otherwise.

The Chernoff Bound For any $0 \leq t \leq n p$ :

$$
\operatorname{Pr}(|B I N(n, p)-n p|>t)<2 e^{-\frac{t^{2}}{3 n p}} .
$$

\section{$4 \quad$ Proof of Lemma 3.3}

It is useful to consider what STRIP looks like from the perspective of $G_{1}$. At each iteration, $G_{1}$ removes all its small components, then is given a list $T_{i}$ of vertices and removes all remaining vertices which are not in $T_{i}$. The following observation about $T_{i}$ is crucial to our analysis: 
Observation 4.1 Given $V_{i}$ and $t_{i}=\left|T_{i}\right|$, the set $T_{i}$ is a uniformly random set of $t_{i}$ vertices from $V_{i}$, and the choice of these vertices is independent of $G_{1}$.

Proof At each step $j<i$ of STRIP, we expose the vertex sets $S_{j}, T_{j}$ without exposing the edges. This implies that for any potential graph $H=G_{2} \cap V_{i}$, every graph obtained by permuting the vertices of $H$ is equally likely to be $G_{2} \cap V_{i}$, and this holds even after conditioning on $G_{1}$ being equal to any particular graph. So consider any two vertex sets $T, T^{\prime} \subset V_{i}$, both of size $t_{i}$, and any graph $H$ such that if $G_{2} \cap V_{i}=H$ then $\left|T_{i}\right|=t_{i}$. By symmetry, the number of ways to permute the vertices of $H$ to obtain a new graph for which $T_{i}=T$ is equal to the number of ways to permute the vertices of $H$ to obtain $T_{i}=T^{\prime}$ (the number is $t_{i} !\left(\left|V_{i}\right|-t_{i}\right)$ !). Summing over all possibilities for $H$ yields that $\operatorname{Pr}\left(T_{i}=T\right)=\operatorname{Pr}\left(T_{i}=T^{\prime}\right)$, even after conditioning on $G_{1}$. This yields the observation.

This implies that if we are only viewing things from the perspective of $G_{1}$, then at each iteration, instead of exposing $T_{i}$ and then deleting $V_{i} \backslash T_{i}$ from $V_{i}$, we can instead just expose $t_{i}=\left|T_{i}\right|$ and remove $\ell_{i}=\left|V_{i}\right|-t_{i}$ uniformly random vertices from $V_{i}$.

It will be convenient to keep track of an additional set $U_{i} \supseteq V_{i}$. Vertices are removed from $U_{i}$ at the same proportional rate that $G_{2}$ causes vertices to be removed from $V_{i}$. $G_{1}$ does not cause any vertices to be removed from $U_{i}$. This set is useful because it extracts the effect that $G_{2}$ has on $V_{i}$ and hence on $S_{i}$. Moreover, $U_{i}$ is close to being independent of $G_{1}$; close enough to be useful. Very roughly speaking, in the limit as $i \rightarrow \infty, V-U_{i}$ can be thought of as $\Theta_{2}^{\prime}$ from Section 2 .

We specify $U_{i}$ using the following modification of STRIP.

As before, at each iteration $i$, we will have a set of vertices $V_{i} \subset\{1, \ldots, n\}$, and we define $S_{i}$, resp. $T_{i}$, to be the vertex set of the largest component of the subgraph of $G_{1}$, resp. $G_{2}$, induced by $V_{i}$.

Again, we only expose the vertices of $S_{i}, T_{i}$. This time, in order to expose $T_{i}$, we will first expose $\left|T_{i}\right|$ and then choose a uniformly random set of $\left|T_{i}\right|$ vertices of $V_{i}$, making use of Observation 4.1. Equivalently, we expose $\ell_{i}=\left|V_{i} \backslash T_{i}\right|$ and obtain $T_{i}$ by deleting $\ell_{i}$ uniformly random vertices from $V_{i}$. We choose those vertices in an unusual manner, to facilitate the specification of $U_{i+1}$.

\section{STRIP1:}

Initialize $V_{1}=U_{1}=\{1, \ldots, n\}$.

For $i=1$ to $\infty$

Expose the vertices of $S_{i}$.

Expose $\ell_{i}=\left|V_{i} \backslash T_{i}\right|$.

If $S_{i}=V_{i}$ and $\ell_{i}=0$ then HALT SUCCEED

Else

Initialize $L_{i}=\emptyset$.

Repeat $\ell_{i}$ times:

Repeat until we choose a $u \in V_{i}$

Choose a uniformly random vertex $u \in U_{i}$ (without replacement).

Place $u$ into $L_{i}$.

$$
\begin{aligned}
& T_{i}:=V_{i} \backslash L_{i} . \\
& V_{i+1}:=S_{i} \backslash L_{i} . \quad \text { (Note that } S_{i} \backslash L_{i}=S_{i} \cap T_{i} \text {.) }
\end{aligned}
$$




$$
\begin{aligned}
& U_{i+1}:=U_{i} \backslash L_{i} . \\
& \text { If } V_{i+1}=\emptyset \text { then HALT FAIL. }
\end{aligned}
$$

Note that, for each $i$, the $\ell_{i}$ vertices of $L_{i}$ that are in $V_{i}$ are uniformly random members of $V_{i}$. So by Observation 4.1, we can couple the choice of $T_{i}$ in STRIP with the choice of $V_{i} \backslash L_{i}$ in STRIP1. Under this coupling, STRIP and STRIP1 produce the same sets $S_{i}, T_{i}, V_{i}$. More precisely:

We run STRIP and STRIP1 in parallel. At iteration $i$, we first expose the vertex sets $S_{i}=$ $S, T_{i}=T$ for STRIP. Then we carry out iteration $i$ of STRIP1 as follows: We set $\ell_{i}=\left|V_{i} \backslash T\right|$. Each time we select $u \in U_{i}$ we first determine whether $u \in V_{i}$. If $u \in V_{i}$ then we pick $u$ to be a uniformly random member of $T$ (without replacement). If $u \notin V_{i}$ then we pick $u$ to be a uniformly random member of $U_{i} \backslash V_{i}$ (without replacement). Note that, by Observation 4.1, $u$ is a uniformly random member of $U_{i}$ (without replacement) and so this is a valid coupling; i.e. $u$ is chosen with the correct distribution for STRIP1. Note also that this coupling ensures that we select $S_{i}=S, T_{i}=T$ for STRIP1, and so STRIP and STRIP1 produce the same sets.

Intuition: So long as $S_{i}$ has linear size, $S_{i}$ will be the largest component of $G_{1} \cap U_{i}$. The reason is that all vertices in $U_{i} \backslash V_{i}$ were removed from $V_{i}$ because they were not in $S_{j}$ for some $j<i$; i.e. they were in small components of $G_{1} \cap V_{j}$. Inductively, this means that they were in small components of $G_{1} \cap U_{j}$ and hence are in small components of $G_{1} \cap U_{i}$, as $U_{i} \subseteq U_{j}$. Therefore, all vertices in the giant component of $G_{1} \cap U_{i}$ are in $V_{i}$, and so the largest component of $G_{1} \cap U_{i}$ is also the largest component of $G_{1} \cap V_{i}$.

This allows us to analyze $\left|S_{i}\right|$ by instead analyzing the largest component of $G_{1} \cap U_{i}$, which is much easier. To do so, we determine the size of $U_{i}$ :

Lemma 4.2 For any constant $i$, w.h.p. $\left|U_{i}\right|=\rho_{i} n+o(n)$.

Intuition: Suppose that we were to define another set $U_{i}^{\prime}$ which is analagous to $U_{i}$ but from the perspective of $G_{2}$. Note that a simple induction shows $V_{i}=U_{i} \cap U_{i}^{\prime}$ : Indeed, every vertex in $V_{i} \backslash T_{i}$ is in $L_{i}$ and hence is not in $U_{i+1}$; similarly every vertex in $V_{i} \backslash S_{i}$ is not in $U_{i+1}^{\prime}$. Since $V_{i+1}=S_{i} \cap T_{i}$ and $V_{i+1} \subseteq U_{i}, U_{i}^{\prime}$, we have $V_{i+1}=U_{i} \cap U_{i}^{\prime}$. By symmetry, $\left|U_{i}^{\prime}\right|=\rho_{i} n+o(n)$. Now $U_{i}$ and $U_{i}^{\prime}$ are not independent, but they are very close - close enough that we have $\left|U_{i} \cap U_{i}^{\prime}\right|=\rho_{i}^{2} n+o(n)$. This is why $\left|V_{i}\right|=\rho_{i}^{2}+o(n)$ (Lemma 3.3).

Proof of Lemmas 3.3, 4.2: We analyze STRIP1; by our coupling, this suffices to prove Lemma 4.2.

We will prove the lemmas by induction. More specifically, we prove there are two sequences of constants $\eta_{1}, \eta_{2}, \ldots$ and $\eta_{1}^{\prime}, \eta_{2}^{\prime}, \ldots$ such that w.h.p.

$$
\begin{aligned}
\left|S_{i}\right|,\left|T_{i}\right| & \in \gamma_{i} n \pm \eta_{i} n^{2 / 3} \\
\left|V_{i}\right| & \in \rho_{i}^{2} n \pm \eta_{i}^{\prime} n^{2 / 3} \\
\left|U_{i}\right| & \in \rho_{i} n \pm \eta_{i}^{\prime} n^{2 / 3} .
\end{aligned}
$$

We will also prove:

Every $v \in U_{i} \backslash V_{i}$ is in a component of size $o(n)$ in $G_{1} \cap U_{i}$ 
We start with $\eta_{1}^{\prime}=0 ; \eta_{1}$ will be implicitly defined below. Our base cases are that $(6),(7)$ and (8) hold trivially for $i=1$. Now we proceed by induction.

Suppose that $V_{i}, S_{i}, \ell_{i}$ are chosen. $S_{i} \subseteq V_{i}$ and $L_{i} \cap V_{i}$ is a set of $\ell_{i}$ uniformly random vertices of $V_{i}$, so $\mathbf{E}\left(\left|S_{i} \cap L_{i}\right|\right)=\frac{\left|S_{i}\right| \ell_{i}}{\left|V_{i}\right|}$. Since $V_{i+1}=S_{i} \backslash L_{i}$, we have

$$
\mathbf{E}\left(\left|V_{i+1}\right|\right)=\left|S_{i}\right|\left(1-\frac{\ell_{i}}{\left|V_{i}\right|}\right)=\frac{\left|T_{i}\right|\left|S_{i}\right|}{\left|V_{i}\right|}
$$

which is in the range $\frac{\gamma_{i}^{2}}{\rho_{i}^{2}} n \pm \frac{1}{2} \eta_{i+1}^{\prime} n^{2 / 3}$, for sufficiently large $\eta_{i+1}^{\prime}$ so long as (5) and (6) hold for $i$.

For any $0 \leq a \leq\left|S_{i}\right|$, the probability that $\left|V_{i+1}\right|=\left|S_{i}\right|-a$ is

$$
\left(\begin{array}{c}
\left|S_{i}\right| \\
a
\end{array}\right)\left(\begin{array}{c}
\left|V_{i}\right|-\left|S_{i}\right| \\
\ell_{i}-a
\end{array}\right) /\left(\begin{array}{c}
\left|V_{i}\right| \\
\ell_{i}
\end{array}\right) .
$$

From this, it is straightforward to show that w.h.p. $\left|V_{i}\right|$ is within $\frac{1}{2} \eta_{i}^{\prime} n^{2 / 3}$ of its mean. Recalling $\rho_{i+1}=\frac{\gamma_{i}}{\rho_{i}}$, this establishes that w.h.p. $\left|V_{i+1}\right| \in \rho_{i+1}^{2} n \pm \eta_{i+1}^{\prime} n^{2 / 3}$; i.e. this yields (6) for $i+1$.

The induction step for $U_{i+1}$ is similar. This time, we have $\mathbf{E}\left(\left|U_{i+1}\right|\right)=\frac{\left|T_{i}\right|\left|U_{i}\right|}{\left|V_{i}\right|}$ which is in the range $\frac{\gamma_{i}}{\rho_{i}} n \pm \frac{1}{2} \eta_{i+1}^{\prime} n^{2 / 3}$ for sufficiently large $\eta_{i+1}^{\prime}$, so long as (5), (6) and (7) hold for $i$.

$\left|U_{i+1}\right|$ is determined by the number of uniformly random vertices of $U_{i}$ that we select for $L_{i}$ before selecting $\ell_{i}$ from $V_{i}$. The probability that this number is $a$ is

$$
\frac{\ell_{i}}{a}\left(\begin{array}{c}
\left|V_{i}\right| \\
\ell_{i}
\end{array}\right)\left(\begin{array}{c}
\left|U_{i}\right|-\left|V_{i}\right| \\
a-\ell_{i}
\end{array}\right) /\left(\begin{array}{c}
\left|U_{i}\right| \\
a
\end{array}\right) .
$$

Explanation: consider the first $a$ vertices selected for $L_{i}$. The event occurs iff (i) exactly $\ell_{i}$ of them are from $V_{i}$ and (ii) one of the vertices from $V_{i}$ is the $a$ th vertex removed.

Again, from this it is straightforward to show that w.h.p. $\left|U_{i}\right|$ is within $\frac{1}{2} \eta_{i}^{\prime} n^{2 / 3}$ of its mean. Recalling $\rho_{i+1}=\frac{\gamma_{i}}{\rho_{i}}$, this establishes that w.h.p. $\left|U_{i+1}\right| \in \rho_{i+1} n \pm \eta_{i+1}^{\prime} n^{2 / 3}$; i.e. this yields (7) for $i+1$.

Suppose that (8) holds for $i$. We will argue below that this implies that $S_{i}$ is the largest component of $G_{1} \cap U_{i}$, so long as that component has linear size; we will focus on the latter component. It would be convenient if $G_{1} \cap U_{i}$ were distributed like $G_{n^{\prime}, p=c / n}$ where $n^{\prime}=\left|U_{i}\right|$. But it is not, since there is some dependency between $G_{1}$ and $\left|U_{i}\right|$. So instead, we sandwich $G_{1} \cap U_{i}$ between two graphs which really are from the $G_{n, p}$ model.

Suppose that (7) holds for $i$. We consider two sets of vertices $U^{-}, U^{+}$, which are defined to be uniformly random subsets of $\{1, \ldots, n\}$ of sizes $x^{-}=\left\lfloor\rho_{i} n-2 \eta_{i}^{\prime} n^{2 / 3}\right\rfloor, x^{+}=\left\lceil\rho_{i} n+2 \eta_{i}^{\prime} n^{2 / 3}\right\rceil$. We couple these two sets with our process as follows:

Choose a sequence $\mathcal{A}=a_{1}, \ldots, a_{n-x^{-}}$uniformly random vertices from $\{1, \ldots, n\}$, without replacement. Set $U^{-}$to be the set of $x^{-}$vertices that are not in $\mathcal{A}$. Set $U^{+}$to be the set of $x^{+}$vertices that are not amongst the first $n-x^{+}$members of $\mathcal{A}$. Thus $U^{-}$is a uniformly random set of $x^{-}$ vertices, $U^{+}$is a uniformly random set of $x^{+}$vertices, and $U^{-} \subset U^{+}$. During the first $i$ iterations of STRIP1, each time we choose a uniform member of $U_{j}, j<i$ to place into $L_{j}$, we simply take the 
next member of $\mathcal{A}$; this is permissable unless the total number of such selections exceeds $n-x^{-}$, i.e. unless $\left|U_{i}\right|<x^{-}$. Note that this chooses the member of $U_{j}$ with the correct (i.e. uniform) distribution.

If $x^{-} \leq\left|U_{i}\right| \leq x^{+}$, then our coupling succeeds and $U^{-} \subseteq U_{i} \subseteq U^{+}$; by our induction hypothesis, this is indeed the case w.h.p. and so we can assume that the coupling succeeds.

Because the vertices of $U^{-}$are selected uniformly at random, we can select them before exposing the edges of $G_{1} \cap U^{-}$. Therefore, we see that $G_{1} \cap U^{-}, G_{1} \cap U^{+}$are distributed as $G_{x^{-}, p=c / n}, G_{x^{+}, p=c / n}$.

Case 1: $\rho_{i}>1 / c$. Let $S^{-}, S^{+}$be the giant components of $G_{1} \cap U^{-}, G_{1} \cap U^{+}$respectively; our coupling implies $S^{-} \subseteq S^{+}$. We argue that w.h.p. $S^{-} \subseteq S_{i} \subseteq S^{+}$, which will easily yield (5) for $S_{i}$. First we analyze the sizes of $S^{-}, S^{+}$.

Lemma 3.4 yields w.h.p. $\left|S^{-}\right| \in \beta n \pm n^{3 / 5}$ where $\beta$ is the positive solution to

$$
\beta=\tau\left(1-e^{-\beta c}\right), \quad \text { with } \tau=x^{-} / n=\rho_{i}-2 \eta_{i}^{\prime} n^{-1 / 3} .
$$

Recalling that $\gamma_{i}$ is the positive solution to $\gamma_{i}=\rho_{i}\left(1-e^{-\gamma_{i} c}\right)$, we see that for $\eta_{i}$ sufficiently large (in terms of $\eta_{i}^{\prime}$ and the value of $\frac{\partial}{\partial \tau} \beta$ at $\tau=\rho_{i}$ ), we have $\beta \geq \gamma_{i}-\frac{1}{2} \eta_{i} n^{-1 / 3}$ and so w.h.p. $\left|S^{-}\right| \geq \gamma_{i} n-\eta_{i} n^{2 / 3}$. Similarly, we obtain that w.h.p. $\left|S^{+}\right| \leq \gamma_{i} n+\eta_{i} n^{2 / 3}$.

Next we argue that w.h.p. $S^{-} \subseteq S_{i} \subseteq S^{+}$. By our coupling, $S^{-}$is contained in a component $Q$ of $G_{1} \cap U_{i}$. We showed above that w.h.p. $S^{-}$has linear size. So, assuming that (8) holds for $i$, all vertices of $Q$ must lie in $V_{i}$, and hence $Q$ is a component of $G_{1} \cap V_{i}$. Since $V_{i} \subseteq U_{i} \subseteq U^{+}$, all vertices of every other component $X$ of $G_{1} \cap V_{i}$ lie in components of $G_{1} \cap U^{+}$. If $X$ does not lie in $S^{+}$then w.h.p. $X$ lies in a component of size $o(n)$ in $G_{1} \cap U^{+}$. If $X$ does lie in $S^{+}$then $X$ has size at most $\left|S^{+}\right|-|Q| \leq\left|S^{+}\right|-\left|S^{-}\right|=o(n)$. Therefore w.h.p. every component of $G_{1} \cap V_{i}$ other than $Q$ has size $o(n)$ and so $S_{i}=Q$. This yields w.h.p. $S^{-} \subseteq S_{i} \subseteq S^{+}$, and hence (5) for $S_{i}$. The proof of (5) for $T_{i}$ follows by symmetry.

We have also shown that every vertex in $U_{i} \backslash S_{i}$ lies in a component of size $o(n)$ in $G_{1} \cap U_{i}$ and hence must lie in a component of size $o(n)$ in $G_{1} \cap U_{i+1}$. Since $U_{i+1} \backslash V_{i+1} \subseteq U_{i} \backslash S_{i}$, this establishes (8) for $i+1$.

Case 2: $\rho_{i}<1 /$ c. In this case, $\gamma_{i}=0$. W.h.p. all components of $G_{x^{+}, p=c / n}$ have size $O(\log n)[5]$. Since $V_{i} \subseteq U_{i} \subset U^{+}$, it follows that w.h.p. every component of $G_{1} \cap V_{i}$ also has size $O(\log n)$ and so $\left|S_{i}\right|=O(\log n)<\eta_{i} n^{2 / 3}$ for any $\eta_{i}$ (and large $n$ ). The same bound holds w.h.p. for $\left|T_{i}\right|$ by symmetry, thus yielding (5) for $i$.

Since $U_{i+1} \subseteq U_{i} \subseteq U^{+}$, every component of $G_{1} \cap U_{i+1}$ also has size $O(\log n)$. This yields (8) for $i+1$.

Case 3: $\rho_{i}=1 /$ c. In this case, again we have $\gamma_{i}=0$. Note that $p=\frac{1}{x^{+}}+O\left(\left(x^{+}\right)^{-4 / 3}\right)$. It is wellknown then that the largest component of $G_{x^{+}, p=c / n}$ w.h.p. has size $\Theta\left(\left(x^{+}\right)^{2 / 3}\right)=\Theta\left(n^{2 / 3}\right)[2,10,8]$. The rest follows as in Case 2, yielding (5) for $i$ and (8) for $i+1$.

To recap, we have shown that the bounds hold:

- (7) and (8) for $i$ imply that w.h.p. (5) holds for $i$ and (8) holds for $i+1$; 
- (5), (6) and (7) for $i$ imply that w.h.p. (6) and (7) hold for $i+1$.

Recall that (6), (7) and (8) hold trivially for $i=1$. For any constant $i$, this induction requires that $O(i)$ events hold where each such event holds w.h.p. Since $i$ is a constant, the union of those events holds w.h.p. This completes the induction and hence proves Lemma 3.3(a,b) and Lemma 4.2.

We close by noting that the arguments above imply Lemma 3.3(c). In particular, we showed that w.h.p. every vertex of $V_{i} \backslash S_{i}$ lies in a component of size $o(n)$ in $G_{1} \cap U^{+}$and hence lies in a component of size $o(n)$ in $G_{1} \cap V_{i}$. By symmetry, the same argument shows that w.h.p. every vertex of $V_{i} \backslash T_{i}$ lies in a component of size $o(n)$ in $G_{2} \cap V_{i}$. Since $V_{i+1}=S_{i} \cap T_{i}$, this yields Lemma 3.3(c).

\section{$5 \quad$ Proof of Theorem 1.1}

Proof of Theorem 1.1(a): Suppose $c<c^{*}$. By Lemma 3.2 we can take $I$ large enough that $\rho_{I}^{2}=0$; thus w.h.p. $\left|V_{I}\right|=o(n)$.

By Lemma 3.3(c), every vertex $v \in V_{1} \backslash V_{2}$ lies in a component of either $G_{1}$ or $G_{2}$ which has size less than $\phi n$, where $\phi=\phi(c)$ comes from Lemma 3.1. Thus Lemma 3.1 implies that $v$ cannot lie in any non-trivial doubly connected component. The same argument, applied inductively, shows that no vertex in $V_{j} \backslash V_{j+1}$ can lie in a non-trivial doubly connected component, for $j<I$. W.h.p. $\left|V_{I}\right|=o(n)<\phi n$ and so no vertex of $V_{I}$ can lie in a non-trivial doubly connected component. This yields Theorem 1.1(a).

To prove part (b), we will run STRIP1 until some large constant $I$ so that $\left|V_{I}\right|,\left|S_{I}\right|$ are very close to their limits $\alpha$, and $\left|U_{I}\right|$ is very close to its limit $\sqrt{\alpha}$. From this point on, Lemma 3.5 implies that each time we delete a vertex $u$ from $U_{i}$, we expect to remove fewer than $2 \sqrt{\alpha}$ vertices from the giant component, $S_{i}$. Note that the probability that $u$ itself is in $S_{i}$ is $\frac{\left|S_{i}\right|}{\left|U_{i}\right|} \approx \sqrt{\alpha}$. So each time we delete a vertex from $S_{i}$ we expect to reduce the size of $S_{i}$ by less than 2 ; i.e. the expected number of additional vertices to be removed from $S_{i}$ is less than one. Because $S_{i}, V_{i}$ are almost the same size, each time we delete a vertex from $V_{i}$, we expect to cause $\left|V_{i} \backslash S_{i}\right|$ to increase by less than one.

It follows that w.h.p. STRIP1 will halt very soon. We can view the vertices of $V_{i} \backslash T_{i}$ as a queue of vertices that must be removed from $G_{1}$ and the vertices of $V_{i} \backslash S_{i}$ as a queue of vertices that must be removed from $G_{2}$. Each time we process a vertex from one queue, it results in an expected increase in the other queue of less than one. So the total size of these queues has a negative drift and with high probability the queues empty quickly. Forthwith the details.

Inspired in part by the approach in [6], we consider a process that, after we find a doubly connected set, continues to remove vertices from $U_{i}\left(S_{i}, T_{i}, V_{i}\right.$ will remain unchanged). We will then consider an iteration that is greater than the time we expect STRIP1 to halt and show that we must indeed have found a doubly connected set before that iteration. Allowing ourselves to consider an iteration that is significantly larger than the actual stopping time of STRIP1 makes this task easier.

As before, at each iteration $i$, we will have a set of vertices $V_{i} \subset\{1, \ldots, n\}$, and we define $S_{i}$, resp. $T_{i}$, to be the vertex set of the largest component of the subgraph of $G_{1}$, resp. $G_{2}$, induced by 
$V_{i}$

\section{KEEP-STRIPPING:}

Initialize $V_{1}=U_{1}=\{1, \ldots, n\}$.

For $i=1$ to $\infty$

Expose the vertices of $S_{i}$.

Expose $\ell_{i}=\left|V_{i} \backslash T_{i}\right|$.

If $S_{i}=V_{i}$ and $\ell_{i}=0$ then

(*) Repeat until $U_{i}=\emptyset$ :

Pick a uniform vertex $u \in U_{i}$

$L_{i}:=\{u\}$

$U_{i+1}:=U_{i} \backslash L_{i}$

$S_{i+1}:=S_{i} ; T_{i+1}:=T_{i} ; V_{i+1}:=V_{i} \quad$ and so these sets remain unchanged.

$i:=i+1$.

Else

Initialize $L_{i}=\emptyset$.

Repeat $\ell_{i}$ times:

Repeat until we choose a $u \in V_{i}$

Choose a uniformly random vertex $u \in U_{i}$ (without replacement).

Place $u$ into $L_{i}$.

$T_{i}:=V_{i} \backslash L_{i}$.

$V_{i+1}:=S_{i} \backslash L_{i}$.

$U_{i+1}:=U_{i} \backslash L_{i}$.

If $V_{i+1}=\emptyset$ then HALT FAIL.

So once the procedure enters $\left(^{*}\right)$, we simply remove vertices from $U_{i}$ one-at-a-time. $S_{i}, T_{i}, V_{i}$ remain unchanged, and because we entered $(*)$, we have $S_{i}=T_{i}=V_{i}$.

Lemma 5.1 For any $c>c^{*}$ and $\delta>0$, there exist constants $I=I(c, \delta)$ such that w.h.p.

(a) $\alpha n<\left|V_{I}\right|<(\alpha+\delta) n$;

(b) STRIP halts after removing at most $\delta n+o(n)$ vertices from $V_{I}$.

Proof Note that to prove (b), it suffices to prove that w.h.p. KEEP-STRIPPING enters $(*)$ within $I+\delta n+o(n)$ iterations. This is because (i) KEEP-STRIPPING is identical to STRIP1 up until the point that STRIP1 halts and KEEP-STRIPPING enters (*); and (ii) STRIP1 and STRIP can be coupled to produce the same sets $S_{i}, T_{i}, V_{i}$.

Recall from Lemma 3.4 that if $\tau c>1$ then w.h.p. the size of the largest component in $G_{n^{\prime}, p=c / n}$ where $n^{\prime}=\tau n$ is in $\beta n \pm n^{3 / 5}$ where $\beta=\beta(\tau)$ is the solution to $\beta=\tau\left(1-e^{-\beta c}\right)$. By Lemma 3.5 and the continuity of $\frac{\partial}{\partial \tau} \beta$, there exists $\xi, \zeta>0$ such that:

$$
\forall \tau \in[\sqrt{\alpha}-\xi, \sqrt{\alpha}+\xi], \text { we have } \frac{\partial}{\partial \tau} \beta<(2-\zeta) \sqrt{\alpha} .
$$


We can assume that $\delta<\xi$ and is sufficiently small in terms of $\zeta$. By Lemma 3.2, we can choose constant $I$ so that $\sqrt{\alpha} \leq \rho_{I}<\sqrt{\alpha}+\frac{\delta}{2}, \alpha \leq \gamma_{I}<\alpha+\frac{\delta}{2}, \rho_{I-1}-\rho_{I}<\delta^{2}$ and $\gamma_{I-1}-\gamma_{I}<\delta^{2}$. By Lemmas 3.3 and 4.2, we have that w.h.p. $\left|U_{I}\right|=\rho_{I} n+o(n),\left|V_{I}\right|=\rho_{I}^{2} n+o(n)$ and both $\left|S_{I}\right|,\left|T_{I}\right|=\gamma_{I} n+o(n)$.

We define $U^{*}$ to be equal to $U_{i}$ at the time where $U_{i}$ has size exactly $\left(\rho_{I}-\delta\right) n$. We have to be careful what we mean by this, since all the vertices in $L_{i}$ are removed from $U_{i}$ at once. So:

Definition 5.2 If, at any point during any iteration $i,\left|L_{i}\right|=\left\lfloor\left(\rho_{I}-\delta\right) n\right\rfloor-\left|U_{i}\right|$ then we set:

- $L^{*}$ is the set of vertices that are in $L_{i}$ at that point;

- $U^{*}=U_{i} \backslash L_{i}$.

- $S^{*}$ is the set of vertices in the largest component of $G_{1} \cap U^{*}$.

We let $J$ denote the iteration during which $U^{*}$ is formed. By Lemma 3.3, w.h.p. $J \geq I$.

We will see that $S^{*}$ has linear size. Note that this implies $S^{*} \subseteq S_{I}$. This is because, as argued in the proof of Lemmas 3.3 and $4.2, S_{I}$ is the largest component of $G_{1} \cap U_{I}$, and every other component in $G_{1} \cap U_{I}$ has size $o(n)$. Since $U^{*} \subset U_{I}$, every component of $G_{1} \cap U^{*}$ that is not contained in $S_{I}$ has size $o(n)$. Therefore if $S^{*}$ has linear size then $S^{*} \subseteq S_{I}$.

Throughout all iterations of KEEP-STRIPPING, each vertex of $L_{i}$ was selected uniformly from $U_{i}$. Therefore, $U^{*}$ is a uniformly random set of $\left\lfloor\left(\rho_{I}-\delta\right) n\right\rfloor$ vertices from $U_{1}=\{1, \ldots, n\}$. Thus, we can expose the subgraph of $G_{1}$ induced by $U^{*}$ by first choosing the vertices of $U^{*}$ and then choosing the edges; i.e. we can treat it as $G_{n^{\prime}=\left\lfloor\left(\rho_{I}-\delta\right) n\right\rfloor, p=c / n}$.

We will prove that w.h.p. we enter line $\left(^{*}\right)$ before forming $U^{*}$; i.e. that w.h.p. we find a doubly connected set before removing $\delta n+o(n)$ vertices from $U_{I}$. So suppose that we form $U^{*}$ before entering line $\left(^{*}\right)$; we will reach a contradiction by bounding $\left|S_{I-1} \backslash S^{*}\right|$ in two different ways.

Since $\alpha(c)$ is increasing with $c, \rho_{I} \geq \sqrt{\alpha(c)}$, and $c>c^{*}$, we have $c \rho_{I}>c^{*} \sqrt{\alpha\left(c^{*}\right)}=(2.4554 \ldots) \times$ $(.5116 . .)^{1 / 2}>1$. So by taking $\delta>0$ sufficiently small, we have $c\left(\rho_{I}-\delta\right)>1$, and so Lemma 3.4 yields that w.h.p. $\left|S^{*}\right|=g n+o(n)$ where $g=\left(\rho_{I}-\delta\right)\left(1-e^{-g c}\right)$.

Since $\delta<\xi$, and $\rho_{I-1}<\rho_{I}+\delta^{2}<\sqrt{\alpha}+\frac{\delta}{2}+\delta^{2}$, we have $\sqrt{\alpha} \leq \rho_{I-1} \leq \sqrt{\alpha}+\xi$. So (9) yields:

$g \geq \gamma_{I-1}-(2-\zeta) \sqrt{\alpha}\left(\rho_{I-1}-\left(\rho_{I}-\delta\right)\right)+o(1)>\gamma_{I-1}-(2-\zeta) \sqrt{\alpha}\left(\delta+\delta^{2}\right)+o(1)>\gamma_{I-1}-\left(2-\frac{2}{3} \zeta\right) \sqrt{\alpha} \delta$,

for $\delta$ sufficiently small in terms of $\zeta$. W.h.p., $\left|S_{I-1}\right|=\gamma_{I-1} n+o(n)$. Therefore w.h.p.

$$
\left|S^{*}\right|=g n+o(n) \geq\left|S_{I-1}\right|-\left(2-\frac{\zeta}{2}\right) \sqrt{\alpha} \delta n .
$$

Consider the set

$$
A=\left(\cup_{i=I}^{J-1} L_{i} \cap V_{i}\right) \cup\left(L^{*} \cap V_{J}\right) .
$$

Note that $A \subseteq S_{I-1} \backslash S^{*}$ since: (a) for each $i \geq I$, we have $V_{i} \subseteq V_{I}$, and so $A \subseteq V_{I} \subseteq S_{I-1}$; (b) $\left(L^{*} \cup_{i=I}^{J-1} L_{i}\right) \cap U^{*}=\emptyset$ so $A \cap S^{*}=\emptyset$. 
Note that $\left|\left(\cup_{i=I}^{J-1} L_{i}\right) \cup L^{*}\right|=\left|U_{I}\right|-\left|U^{*}\right|=\delta n+o(n)$. The vertices of $L_{i}$ are selected uniformly from $U_{i}$. Since $\left|V_{i}\right| \geq\left|V_{I}\right|-\delta n$, each choice is a member of $V_{i}$ with probability at least

$$
\frac{V_{I}-\delta n+o(n)}{U_{I}}=\frac{\left(\rho_{I}^{2}-\delta\right) n+o(n)}{\rho_{I} n+o(n)}>\rho_{I}-\frac{2 \delta}{\rho_{I}}>\sqrt{\alpha}-\frac{2 \delta}{\sqrt{\alpha}},
$$

since $\rho_{I}>\sqrt{\alpha}$. So the number of these vertices that are in $V_{i}$ is dominated from below by the binomial variable $B I N\left(\delta n+o(n), \sqrt{\alpha}-\frac{2 \delta}{\sqrt{\alpha}}\right)$. The Chernoff Bound implies that w.h.p.

$$
|A| \geq \delta\left(\sqrt{\alpha}-\frac{3 \delta}{\sqrt{\alpha}}\right) n
$$

Recall that STRIP1, KEEP-STRIPPING and $U_{i}$ were based on viewing STRIP from the perspective of $G_{1}$. Now consider defining analogous procedures that view it from the perspective of $G_{2}$; i.e. we define $L_{i}^{\prime}, U_{i}^{\prime},\left(L^{*}\right)^{\prime},\left(U^{*}\right)^{\prime}, T^{*}$, and KEEP-STRIPPING ${ }^{\prime}$ by replacing $G_{1}$ by $G_{2}$ throughout the definitions of $U_{i}$, STRIP1 and KEEP-STRIPPING. We define $J^{\prime}$ to be the analogue of $J$.

We couple KEEP-STRIPPING and KEEP-STRIPPING'. Thus, they run in parallel and produce the same sets $S_{i}, T_{i}, V_{i}$. To be specific, note that we enter $\left(^{*}\right)$ during the same iteration in KEEP-STRIPPING and KEEP-STRIPPING'. Until that iteration, both these procedures are coupled with STRIP in the same way that STRIP1 is coupled with STRIP; thus they are coupled together. After entering $(*)$, they are decoupled and $U_{i}, U_{i}^{\prime}$ evolve independently.

By symmetry, the same proof used for (10) yields that w.h.p.

$$
\left|T^{*}\right| \geq\left|T_{I-1}\right|-\left(2-\frac{\zeta}{2}\right) \sqrt{\alpha} \delta n
$$

We define

$$
A^{\prime}=\left(\cup_{i=I}^{J^{\prime}-1} L_{i}^{\prime} \cap V_{i}\right) \cup\left(\left(L^{*}\right)^{\prime} \cap V_{J^{\prime}}\right) .
$$

By symmetry, the same proof used for (11) yields that w.h.p.

$$
\left|A^{\prime}\right| \geq \delta\left(\sqrt{\alpha}-\frac{3 \delta}{\sqrt{\alpha}}\right) n
$$

Note that if $J \geq J^{\prime}$ and we do not enter $(*)$ before iteration $J$ then $A^{\prime} \subseteq S_{I-1} \backslash S^{*}$. This is because: (a) $A^{\prime} \subseteq V_{I} \subseteq S_{I-1}$ for the same reason that $A \subseteq V_{I}$ above. (b) For each iteration $i$ before entering $\left(^{*}\right), S_{i}=V_{i} \backslash L_{i}^{\prime}$, just as $T_{i}=V_{i} \backslash L_{i}$. So every vertex in $A^{\prime}$ is in $V_{i} \backslash S_{i}$ for some $I \leq i \leq J^{\prime} \leq J$ and hence is not in $S_{J} \supseteq S^{*}$

Similarly, if $J \leq J^{\prime}$ and we do not enter (*) before iteration $J^{\prime}$ then $A, A^{\prime} \subseteq T_{I-1} \backslash\left(S^{*}\right)^{\prime}$.

Finally, we wish to bound the number of vertices in $A \cap A^{\prime}$. If $J \geq J^{\prime}$ and we do not enter (*) before iteration $J$, then we argued above that $A^{\prime} \subseteq \cup_{i=I}^{J} V_{i} \backslash S_{i}$. It follows that every member of $A \cap A^{\prime}$ is a member of $L_{i} \cap\left(V_{i} \backslash S_{i}\right)$ for some $I \leq i \leq J-1$ or $L^{*} \cap\left(V_{J} \backslash S_{J}\right)$.

Similarly, if $J \leq J^{\prime}$ and we do not enter $\left(^{*}\right)$ before iteration $J^{\prime}$, then every member of $A \cup A^{\prime}$ is a member of $L_{i}^{\prime} \cap\left(V_{i} \backslash T_{i}\right)$ for some $I \leq i \leq J^{\prime}-1$ or $\left(L^{*}\right)^{\prime} \cap\left(V_{J^{\prime}} \backslash T_{J^{\prime}}\right)$. 
Recall that $V_{i} \backslash S_{i} \subseteq S_{I-1} \backslash S^{*}$ for $I \leq i \leq J$. So applying (10), we find that during iteration $i$, each of the $\left|L_{i}\right|$ (or $\left|L^{*}\right|$ if $i=J$ ) vertices chosen from $U_{i}$ is in $V_{i} \backslash S_{i}$ with probability

$$
\frac{\left|V_{i} \backslash S_{i}\right|}{\left|U_{i}\right|}<\frac{\left|S_{I-1} \backslash S_{J}\right|}{\left|U^{*}\right|}<\frac{\left(2-\frac{\zeta}{2}\right) \sqrt{\alpha} \delta n}{\left(\rho_{I}-\delta\right) n}<3 \delta .
$$

So $\left|\left(\cup_{i=I}^{J-1} L_{i} \cap\left(V_{i} \backslash S_{i}\right)\right) \cup\left(L^{*} \cap\left(V_{J} \backslash S_{J}\right)\right)\right|$ is dominated from above by the binomial variable $B I N(\delta n+$ $o(n), 3 \delta)$. So the Chernoff Bound yields that w.h.p.

$$
\left|\left(\cup_{i=I}^{J-1} L_{i} \cap\left(V_{i} \backslash S_{i}\right)\right) \cup\left(L^{*} \cap\left(V_{J} \backslash S_{J}\right)\right)\right| \leq 4 \delta^{2} n .
$$

Similarly, w.h.p.

$$
\left|\left(\cup_{i=I}^{J^{\prime}-1} L_{i}^{\prime} \cap\left(V_{i} \backslash T_{i}\right)\right) \cup\left(\left(L^{*}\right)^{\prime} \cap\left(V_{J^{\prime}} \backslash T_{J^{\prime}}\right)\right)\right| \leq 4 \delta^{2} n .
$$

If $J \geq J^{\prime}$ then $\left|A \cap A^{\prime}\right|$ is bounded by (14), and if $J \leq J^{\prime}$ then $\left|A \cap A^{\prime}\right|$ is bounded by (15). Therefore, w.h.p.

$$
\left|A \cap A^{\prime}\right| \leq 4 \delta^{2} n .
$$

If $J \geq J^{\prime}$ and we do not enter (*) before iteration $J$, then $A \cup A^{\prime} \subseteq S_{I-1} \backslash S^{*}$ and (11), (13), (16) imply that w.h.p.

$$
\left|S^{*}\right| \leq\left|S_{I-1}\right|-2 \times \delta\left(\sqrt{\alpha}-\frac{3 \delta}{\sqrt{\alpha}}\right) n+4 \delta^{2} n<\left|S_{I-1}\right|-\left(2-\frac{\zeta}{4}\right) \sqrt{\alpha} \delta n,
$$

for $\delta$ sufficiently small in terms of $\zeta$, which contradicts $(10)$.

Similarly, if $J \leq J^{\prime}$ and we do not enter $\left(^{*}\right)$ before iteration $J^{\prime}$, then $A \cup A^{\prime} \subseteq T_{I-1} \backslash T^{*}$ and (11), (13), (16) contradict (12).

Thus, w.h.p. KEEP-STRIPPING must enter $\left(^{*}\right)$ before $\max \left(J, J^{\prime}\right)$ iterations, and thus either before $\delta n+o(n)$ vertices are removed from $U_{I}$ or before $\delta n+o(n)$ vertices are removed from $U_{I}^{\prime}$. Either way, this is before $\delta n+o(n)<2 \delta n$ vertices are removed from $V_{I}$. This proves the lemma.

The remaining part of our main theorem follows immediately:

Proof of Theorem 1.1(b): For any $\delta>0$, Lemma 5.1 yields that STRIP will w.h.p. Halt Succeed and produce a set $S=T=V$ of size at most $(\alpha+\delta) n$ and at least $(\alpha-\delta) n-o(n)$. This will be a doubly connected set of $\left(G_{1}, G_{2}\right)$.

Take $\delta<\phi=\phi(c)$ (from Lemma 3.1). The argument from the proof of Theorem 1.1(a) implies that no vertex outside of $V_{I}$ is in a doubly connected set. Since $\left|V_{I} \backslash V\right|<\delta n+o(n)<\phi n$, every vertex in $V_{j} \backslash V_{j+1}, j \geq I$ is in a component of size less than $\phi n$ in either $G_{1} \cap V_{j}$ or $G_{2} \cap V_{j}$. This, Lemma 3.3(c), and the same argument from the proof of Theorem 1.1(a) implies that no vertex outside of $V$ is in a doubly connected set. Therefore $V$ is the largest doubly connected set of $\left(G_{1}, G_{2}\right)$.

Taking $\delta$ to be arbitrarily small yields Theorem 1.1(b). 


\section{Acknowledgement}

We would like to thank anonymous referees for helpful comments on an earlier draft of this paper.

\section{References}

[1] B. Bollobás, Random Graphs. 2nd edition. Cambridge University Press, 2001.

[2] B. Bollobás. The evolution of random graphs. Trans. Am. Math. Soc. 286 (1984), 257 - 274.

[3] B. Bollobás and O. Riordan, Asymptotic normality of the size of the giant component via a random walk. arXiv:1010.4595

[4] H. Chernoff. A measure of asymptotic efficiency for tests of a hypothesis based on the sum of observations. Ann. Math. Statist. 23493 - 509 (1952).

[5] P. Erdős and A. Rényi. On the evolution of random graphs. Magayar Tud. Akad. Mat. Kutato Int. Kozl. 5 (1960), 17 - 61.

[6] D. Fernholz and V. Ramachandran. Cores and Connectivity in Sparse Random Graphs. The University of Texas at Austin, Department of Computer Sciences, technical report TR-04-13 (2004).

[7] S. Janson and M. Luczak. A simple solution to the $k$-core problem. Random Structures Algorithms 30 (2007) 50 - 62 (2007).

[8] S. Janson, T. Łuczak and A. Ruciński. Random Graphs. Wiley, New York (2000).

[9] J.H.Kim. Poisson cloning model for random graphs. arXiv:0805.4133v1

[10] T. Łuczak. Component behaviour near the critical point of the random graph process. Rand. Struc. \& Alg. 1 (1990), 287 - 310.

[11] M. Molloy Cores in random hypergraphs and boolean formulas. Random Structures and Algorithms 27, 124 - 135 (2005).

[12] M. Molloy The freezing threshold for $k$-colourings of a random graph. Proceedings of STOC 2012 .

[13] M. Molloy and B. Reed, Graph colouring and the probabilistic method, Springer-Verlag, Berlin, 2001.

[14] B. Pittel, J. Spencer and N. Wormald, Sudden emergence of a giant $k$-core in a random graph. J.Comb.Th.(B) 67 (1996), 111 - 151.

[15] B. Pittel and N. Wormald, Counting connected graphs inside-out. J. Comb.Th. B 93 (2005), $127-172$.

[16] V.E. Stepanov, Some features of the structure of a random graph near a critical point. (Russian) Teor. Veroyatnost. i Primenen. 32 (1987), 633657. 